\title{
Advantages of additional PET vs. MRI in the clinical diagnosis of anterior mediastinal tumors
}

\author{
TOSHIKI YAJIMA, AKIRA MOGI, EI YAMAKI, RYOUICHI ONOZATO, \\ TAKAYUKI KOSAKA, KEN SHIRABE and HIROYUKI KUWANO \\ Department of General Surgical Science, Gunma University Graduate School of Medicine, \\ Maebashi-shi, Gunma 371-8511, Japan
}

Received April 1, 2019; Accepted August 19, 2020

DOI: $10.3892 / \operatorname{mco} .2020 .2155$

\begin{abstract}
The present study retrospectively examined the diagnostic utility of adding positron emission tomography (PET) or magnetic resonance imaging (MRI) to computed tomography (CT) alone for preoperative diagnosis of anterior mediastinal tumors. A total of 104 consecutive patients who had undergone surgical resection of anterior mediastinal tumors were divided into two groups: Additional PET to another modality and no additional PET to another modality, and further subdivided into three groups: CT alone, additional MRI to CT and additional PET to CT. The sensitivity, specificity, and accuracy for diagnosing malignant tumors in each subgroup was calculated. Comparing the two groups, the diagnostic sensitivity was similar for additional PET (98.0\%) and no additional PET (95.2\%) groups; however, the specificity and accuracy for additional PET (75.0 and 92.2\%, respectively) were significantly improved compared with no additional PET (31.6 and $65.0 \%$, respectively). In the subgroup analysis, adding PET to CT showed an improvement in specificity and positive predictive value for detecting malignant tumors, compared with either additional MRI to CT or CT alone. Additional PET, but not MRI, has advantages over $\mathrm{CT}$ alone in clinically distinguishing benign from malignant tumors of the mediastinum.
\end{abstract}

\section{Introduction}

Anterior mediastinal tumors, including various types of neoplasms, are relatively rare $(1,2)$. Although percutaneous needle biopsy is being used more frequently to obtain a pathological diagnosis, more invasive procedures need to be performed to obtain adequate tissue due to their size, location and heterogeneity and patient characteristics (3-5). Therefore,

Correspondence to: Dr Toshiki Yajima, Department of General Surgical Science, Gunma University Graduate School of Medicine, 3-39-22 Showa-machi, Maebashi-shi, Gunma 371-8511, Japan

E-mail: yajimato@gunma-u.ac.jp

Key words: anterior mediastinal tumors, benign tumor, positron emission tomography, MRI the preoperative diagnosis of anterior mediastinal tumors is comprehensively determined by their clinical presentation and several imaging modalities, including computed tomography $(\mathrm{CT})$, magnetic resonance imaging (MRI) or positron emission tomography (PET). Although essential for the detection of anterior mediastinal tumors, CT alone cannot differentiate between benign and malignant tumors in some cases (5). To improve the accuracy of preoperative diagnosis, MRI and/or PET is added to CT, depending upon the surgeon's discretion following guidelines (5). Aside from tumor detection, MRI is useful in the qualitative assessment of tumors such hemorrhagic or inflammatory thymic cysts that mimic solid tumors $(6,7)$. The utility of PET for the clinical diagnosis of malignant tumors, including thymoma and thymic cancer, has been reported in numerous publications (8-19). Fluorodeoxyglucose (FDG) accumulation in high-risk thymoma, particularly thymic carcinoma, is evidently greater compared with in low-risk thymoma (10-18,20). The role of fluorodeoxyglucose-positron emission tomography (FDG-PET) in differentiating benign and malignant thymic masses has been previously evaluated by several small-scale studies (21). Therefore, the clinical significance of PET in distinguishing malignant from benign anterior mediastinal tumors is still unclear. Furthermore, the diagnostic accuracy of CT for malignant anterior mediastinal tumors may further improve by addition of PET or MRI, but it remains unclear whether either adding PET or MRI is useful. Accurate diagnosis of benign tumors by imaging modalities is important to avoid unnecessary open thoracotomy. The present study aimed to elucidate the functionality of adding a PET or MRI to CT in the diagnosis of the anterior mediastinal tumors, compared with CT alone. The present study retrospectively analyzed the sensitivity, specificity, positive predictive value (PPV) and negative predictive value (NPV) for these modalities.

\section{Patients and methods}

Population. The study was a retrospective analysis of the medical records of 104 consecutive patients (52 males and 52 females; age range, 1-84 years, mean age 54.0 years) who had undergone surgery for anterior mediastinal tumor at the Department of General Surgical Science at Gunma University (Gunma, Japan) between April 2003 and March 2015. Patients 
who had undergone percutaneous biopsy during the same period for a preoperative diagnosis were excluded from the present study. All patients had undergone clinical examination, chest X-rays and CT of the chest; additional PET and/or MRI were determined by each surgeon during the preoperative diagnosis. In the subgroup analysis, 31 patients with both additional PET and MRI to CT were excluded to directly compare with the advantages of additional PET vs. MRI to CT in the clinical diagnosis of anterior mediastinal tumors. It is difficult to determine the effect of adding either PET or MRI on accurate diagnosis in anterior mediastinal tumors in patients with additional PET and MRI. The study was approved by the ethics committee of the Gunma University Hospital.

FDG-PET imaging. Patients fasted for at least $4 \mathrm{~h}$ before ${ }^{18} \mathrm{~F}$-FDG PET examination. Patients received intravenous FDG at a dose of 200-250 million Becquerels (MBq) $\sim 1 \mathrm{~h}$ before imaging. Image acquisition was performed using the Advance NXi PET scanner (GE Healthcare) and the Discovery (LS) PET scanner (GE Healthcare). Three-dimensional emission scanning was performed from the groin area up to the top of the skull. The PET images were reviewed independently by two experienced physicians. The data obtained were reconstructed by iterative ordered subset expectation maximization. To evaluate ${ }^{18} \mathrm{~F}$-FDG accumulation, each tumor was examined visually and the maximum standardized uptake value (SUV) of the entire tumor was determined.

Preoperative diagnosis and data analysis. The radiological findings on CT, MRI or PET were examined by two radiologists from the Gunma University Graduate School of Medicine who specialized in diagnosing mediastinal lesions. Final preoperative diagnosis was comprehensively determined by clinical presentation and several imaging modalities that were discussed in a conference that involved chest radiologists, physicians and thoracic surgeons. Pathological diagnosis was confirmed on surgically resected specimens. Clinical preoperative diagnosis and surgical pathological diagnosis, as well as sensitivity, specificity, PPV, NPV and accuracy were compared among the patient groups. Specifically, patients were divided into two groups: Additional PET to another modality and no additional PET to another modality. The patients were further subdivided into three groups to compare with the advantages of additional PET vs. MRI to CT in the clinical diagnosis of anterior mediastinal tumors: i) $\mathrm{CT}$ alone; ii) additional MRI to $\mathrm{CT}$ and iii) additional PET to $\mathrm{CT}$.

Statistical analysis. McNemar's test was used to compare the sensitivities, specificities and overall accuracies of additional PET or MRI to CT. Student's t-test and $\chi^{2}$ test were used to compare continuous and categorical variables, respectively. Calculations and statistical tests were performed using JMP 5.0 (SAS Institute Inc.). $\mathrm{P}<0.05$ was considered to indicate a statistically significant difference.

\section{Results}

Pathological diagnosis of anterior mediastinal tumors. Among the 104 patients included in the present study,
Table I. Pathological diagnosis of anterior mediastinal tumors after surgical resection.

\begin{tabular}{|c|c|}
\hline Pathological diagnosis & No. $(\%)$ \\
\hline \multicolumn{2}{|l|}{ Benign } \\
\hline Thymic cysts & $18(17.3)$ \\
\hline Mature teratoma & $6 \quad(5.8)$ \\
\hline Bronchogenic cysts & $3(2.9)$ \\
\hline Pericardial cysts & $3(2.9)$ \\
\hline Other & $5(4.8)$ \\
\hline Total (Benign) & $35(33.7)$ \\
\hline \multicolumn{2}{|l|}{ Malignant } \\
\hline Thymoma & $51(49.0)$ \\
\hline Thymic carcinoma & $8 \quad(7.7)$ \\
\hline Thymic carcinoid & $4 \quad(3.8)$ \\
\hline Germinoma & $2(1.9)$ \\
\hline Other & $4(3.8)$ \\
\hline Total (Malignant) & $69(66.3)$ \\
\hline
\end{tabular}

$69(66.3 \%)$ had malignant tumors, including thymoma $(\mathrm{n}=51)$, thymic cancer $(n=8)$, thymic carcinoid $(n=4)$, and germinoma $(n=2)$ (Table I). Two malignant lesions, from the stomach and the thyroid, were of metastatic origin. The remaining 35 patients (33.7\%) had benign lesions, including thymic cysts $(n=18)$, mature teratoma $(n=6)$, bronchogenic cysts $(n=3)$, pericardial cysts $(n=3)$, and others $(n=5)$, such as neurogenic tumors, lymphangioma, fibroinflammatory lesions, cavernous hemangioma and chondroma.

Comparison of clinical factors with or without additional PET to another modality for the detection of anterior mediastinal tumors. Due to increased glucose metabolism, malignant tumors typically demonstrate higher FDG uptake compared with benign tumors (Figs. 1 and 2). The proportion of the patients who underwent imaging modalities with additional PET and those without additional PET for preoperative diagnosis were $62.5 \%(n=65)$ and $37.5 \%(n=39)$, respectively. There was no significant difference in the age, sex, tumor size, preoperative diagnosis and surgical approach in the two subgroups (Table II). The ratio of CT enhancement and presence of MRI images were comparable between the two subgroups. In cases that did not undergo PET, the sensitivity and specificity of the clinical diagnosis of malignancy was 95.2 and $31.6 \%$, respectively (Table III). The sensitivity, specificity, PPV, NPV and accuracy of the additional PET in detecting malignancy were $98.0,75.0,92.2,92.3$ and $92.2 \%$, respectively. There were significant differences in specificity and accuracy for detecting malignancy between the additional PET and the no additional PET group.

Comparison of efficacy of adding PET or MRI to CT for the detection of malignant tumors. To directly compare the advantages of additional PET or MRI to CT in the clinical diagnosis of anterior mediastinal tumors, a subgroup analysis was performed on 73 patients ( 31 patients were 

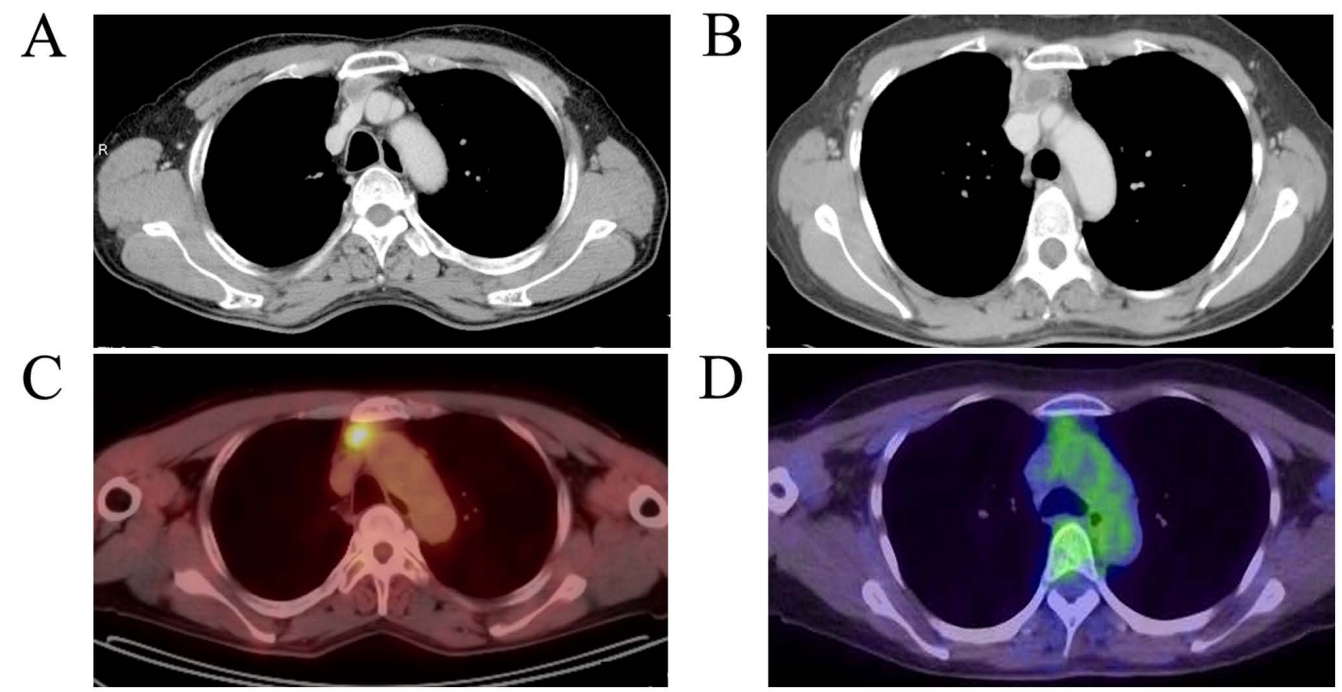

Figure 1. Mediastinal lesions are diagnosed as malignant tumors by adding positron emission tomography due to high fluorodeoxyglucose uptake. (A and B) Mediastinal lesions in a 71-year-old man were incidentally detected by enhanced CT during follow-up for other diseases. Pathological diagnosis revealed the mass to be thymic carcinoma after operation. (C and D) Mediastinal cystic lesions in a 54-year-old female was detected by enhanced CT during follow-up for other diseases. The pathological examination revealed the mass to be a type B2 thymoma.
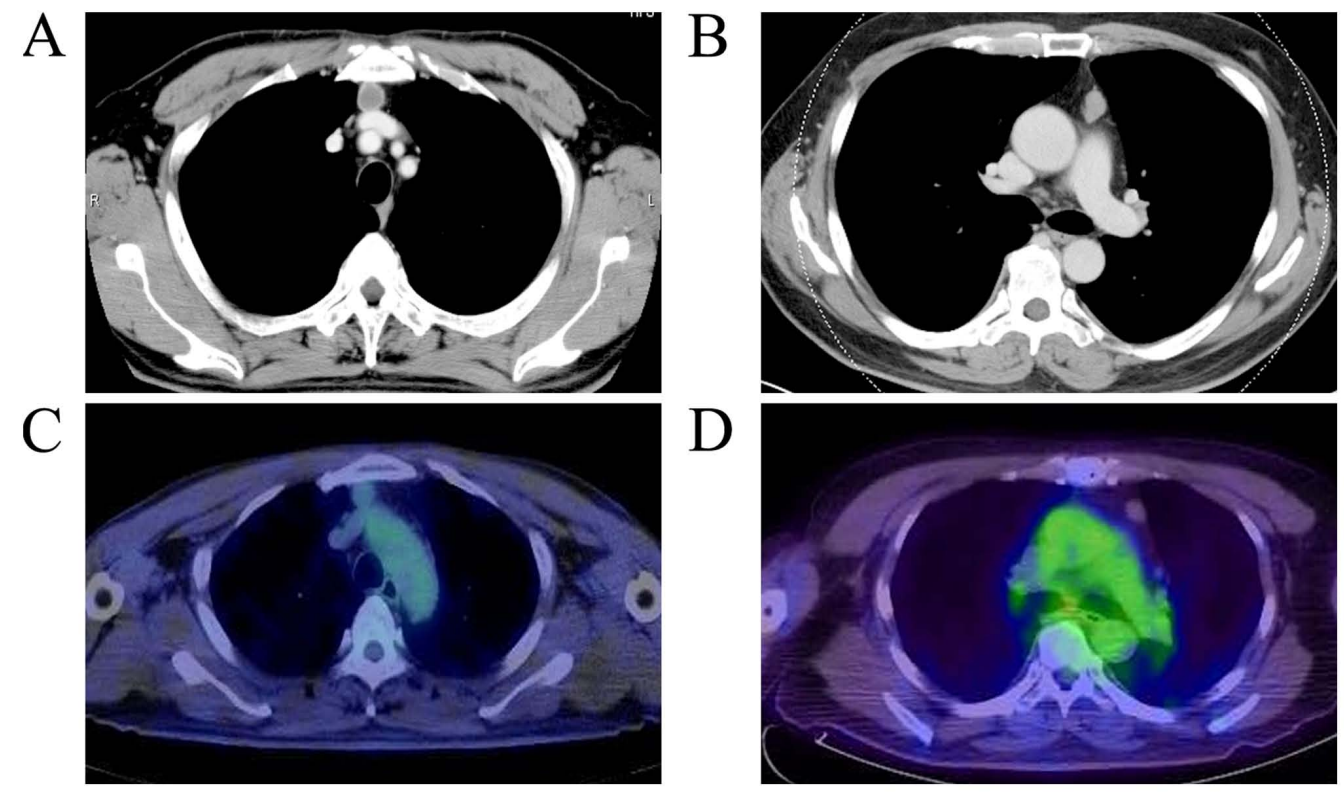

Figure 2. Cases with growing cystic lesions with benign thymic cysts misinterpreted as cystic thymoma by enhanced CT in anterior mediastinal tumors in (A) a 61-year-old male or (B) a 49-year-old female. (C and D) These lesions were correctly interpreted as benign lesions by adding positron emission tomography to $\mathrm{CT}$ as the fluorodeoxyglucose uptake of lesions was not detected. Surgical resections were conducted by patients' preferences.

excluded) with additional PET and MRI to CT. The proportion of the patients who underwent preoperative diagnosis by $\mathrm{CT}$ alone, additional MRI to CT and additional PET to CT were $28.7 \%(n=21), 26.0 \%(n=19)$ and $45.2 \%(n=33)$, respectively (Table SI). To directly compare the efficacy between adding PET and MRI, three subgroups were selected: CT alone, adding MRI to CT and adding PET to CT. As shown in Table IV, CT alone had sufficient diagnostic sensitivity for malignant tumors and there was no additional improvement in sensitivity after adding PET or MRI. However, all seven benign lesions were diagnosed as malignant by $\mathrm{CT}$ alone (Table IV). None of the patients with benign tumors were classified correctly (specificity $=0 \%$ ); in these cases, the specificity and NPV of adding MRI to $\mathrm{CT}$ to detect malignant lesions increased up to 50 and $100 \%$, respectively; the accuracy was comparable with CT alone. Adding PET to CT showed a significant improvement in specificity and accuracy for detecting malignant tumors, compared with either additional MRI to CT or CT alone.

However, the results of the present study are subject to bias due to its retrospective design. Although almost all the clinicopathological factors were comparable among the groups, the mean age of the additional MRI to CT group was lower and had a higher frequency of benign lesions compared with other groups (Table SI). 
Table II. Comparison of clinical factors with or without additional PET to another modality for the detection of anterior mediastinal tumors.

\begin{tabular}{|c|c|c|c|}
\hline Characteristic & PET- $(n=39)$ & $\mathrm{PET}+(\mathrm{n}=65)$ & P-value \\
\hline Age, mean (range) (years) & $50.6(1-84)$ & $56.5(28-79)$ & 0.0776 \\
\hline $\mathrm{M} / \mathrm{F}[\mathrm{n}(\%)]$ & $18 / 21(46.2 / 53.9)$ & $34 / 31(52.3 / 47.7)$ & 0.5433 \\
\hline Tumor size, mean (range) $(\mathrm{cm})$ & $4.3(1.4-9.5)$ & $4.6(1.0-11.5)$ & 0.6530 \\
\hline CT enhancement $-/+[\mathrm{n}(\%)]$ & $9 / 30(23.1 / 76.9)$ & $6 / 59(9.2 / 90.8)$ & 0.0558 \\
\hline Additional MRI $-/+[\mathrm{n}(\%)]$ & $19 / 20(48.7 / 51.3)$ & $33 / 32(50.8 / 49.2)$ & 0.8395 \\
\hline Preoperative diagnosis, benign/malignant $[\mathrm{n}(\%)]$ & $7 / 32(18.0 / 82.0)$ & $13 / 52(20.0 / 80.0)$ & 0.7972 \\
\hline Approach, VATS/open [n (\%)] & $16 / 23(41.1 / 58.9)$ & $29 / 36(44.6 / 55.4)$ & 0.7206 \\
\hline
\end{tabular}

M, male; F, female; VATS, video-assisted thoracoscopic surgery.

Table III. Efficacy comparison of adding PET for the detection of malignant tumors.

\begin{tabular}{|c|c|c|c|c|c|}
\hline Subgroup & Sensitivity & Specificity & PPV & NPV & Accuracy \\
\hline PET- & $95.2(19 / 20)$ & $31.6(6 / 19)$ & $60.6(19 / 32)$ & $85.7(6 / 7)$ & $65.0(25 / 39)$ \\
\hline PET+ & $98.0(48 / 49)$ & $75.0^{\mathrm{a}}(4 / 16)$ & $92.2(48 / 52)$ & $92.3(12 / 13)$ & $92.2^{\mathrm{a}}(60 / 65)$ \\
\hline
\end{tabular}

${ }^{a} \mathrm{P}<0.05$ vs. PET-. PET, positron emission tomography; PPV, positive predictive value; NPV, negative predictive value.

Table IV. Efficacy comparison of adding PET and/or MRI to CT for the detection of malignant tumors.

\begin{tabular}{|c|c|c|c|c|c|}
\hline Subgroup & Sensitivity & Specificity & PPV & NPV & Accuracy \\
\hline CT alone & $92.9(13 / 14)$ & $0(0 / 7)$ & $69.0(13 / 20)$ & $0(0 / 1)$ & $61.9(13 / 21)$ \\
\hline $\mathrm{CT}+\mathrm{MRI}$ & $100(6 / 6)$ & $50.0^{\mathrm{a}}(6 / 12)$ & $50.0(6 / 12)$ & $100(6 / 6)$ & $63.1(12 / 19)$ \\
\hline $\mathrm{CT}+\mathrm{PET}$ & $100(28 / 28)$ & $80.0^{\mathrm{a}, \mathrm{b}}(4 / 5)$ & $96.6(28 / 29)$ & $100(4 / 4)$ & $97.0^{\mathrm{a}, \mathrm{b}}(32 / 33)$ \\
\hline
\end{tabular}

${ }^{\mathrm{a}} \mathrm{P}<0.05$ vs. $\mathrm{CT}$ alone. ${ }^{\mathrm{b}} \mathrm{P}<0.05$ vs. $\mathrm{CT}+\mathrm{MRI}$. PET, positron emission tomography; PPV, positive predictive value; NPV, negative predictive value.

\section{Discussion}

Preoperative diagnosis of anterior mediastinal tumors is comprehensively determined by clinical presentation and several imaging modalities as needle biopsy is usually anatomically difficult to perform. Because there has been no consensus on the optimal preoperative diagnosis of anterior mediastinal tumors, the choice of imaging modality to add to CT is determined by the surgeon (1-5). The present study found the utility of adding PET, but not MRI, to CT for detecting benign from malignant anterior mediastinal tumors.

CT is essential for the diagnosis of anterior mediastinal tumors (5). It was reported that morphological changes, including intralesional fat, midline location and triangular thymic shapes on the CT may help characterize thymic masses as benign $(22,23)$. In the present study, all seven benign lesions were diagnosed as malignant by CT alone, based on the lobulated contour, heterogeneous enhancement and increased diameter. The specificity of CT examination alone was more accurate in all cases, including follow-up cases without surgical indication because our series included only consecutive patients who had undergone surgical resection for highly suspicious malignant lesions. However, in the present study, CT alone had higher sensitivity, but lower specificity in detecting malignancy, indicating that it was not sufficient for the diagnosis of benign anterior mediastinal tumors.

Numerous studies reported the utility of PET for the diagnosis of malignant anterior mediastinal tumors (8-20). Due to increased glucose metabolism, malignant tissues typically demonstrate higher FDG uptake compared with benign and normal tissue. The role of FDG PET in differentiating benign and malignant thymic masses was previously evaluated in a study, albeit a small sample size (21). Therefore, the clinical significance of PET in differentiating benign and malignant anterior mediastinal tumors is still unclear. The present study found that adding PET to CT showed high sensitivity and specificity for detecting these tumors. In particular, the specificity and PPV improved after adding PET, as almost all the benign anterior mediastinal lesions did not have an FDG uptake, resulting in true diagnosis as a benign lesion (Fig. 2). 
The high accuracy of additional PET to CT was not affected by irrespective of MRI presence. Therefore, preoperative diagnosis of anterior mediastinal tumors by $\mathrm{CT}$ requires additional PET, but not MRI. A number of patients may undergo invasive procedures for benign disease; in fact, in the present study, 28 patients underwent resection and 13 patients underwent sternotomy. Additional PET to CT may be valuable in deciding the need for the surgical resection of anterior mediastinal tumors and could be considered as standard procedure in the preoperative assessment of patients with these tumors in the future.

MRI is useful for the qualitative assessment of tumors, especially cystic lesions, including benign thymic cysts (5). Therefore, adding MRI to CT may improve the specificity and false-positive rate of detecting malignant tumors. The present study found that compared with CT alone, adding MRI to CT substantially increased the specificity, but the false-positive rate was higher (CT: 7/7, MRI: 6/12). The proportion of benign lesions detected by additional MRI to CT was significantly higher $(63.2 \%)$ compared with the other groups $(37.1 \%)$; differences in patient characteristics may have contributed to the lower specificity of additional MRI to CT. A study reported that diffusion-weighted (DW) MRI had much higher specificity and accuracy for differentiating between malignant and benign anterior mediastinal lesions than conventional MRI protocols (24). Further analysis is needed to confirm the utility of MRI in the diagnosis of anterior mediastinal tumors.

In addition, it was reported that DW MRI could add a 'histologic' parameter for malignant invasion by measuring cell density and apparent diffusion coefficient (ADC) values (24). Therefore, combining the SUV of PET and the ADC of DW MRI may be more useful in diagnosing malignancy. Further studies are needed to confirm this possibility.

The present study did not assess the utility of each modality for detecting malignant tumors because, in our opinion, a comprehensive diagnosis using several imaging modalities may be more clinically realistic. Clinical presentation, including tumor markers and signs of myasthenia gravis (MG), also play an important role in the preoperative diagnosis of anterior mediastinal tumors (1-5). In the present study, the number of patients diagnosed with thymoma with MG by CT alone was higher than that by adding PET to CT (data not shown). These data showed that additional PET did not improve the specificity and accuracy of CT alone. Several detailed examinations on clinical presentation are needed to establish the utility of PET.

The present study had several limitations. First is the retrospective design with the relatively small number of samples; although $>100$ patients were included, we plan to extend the study to a larger patient population. Since anterior mediastinal tumors are relatively rare, a large scale, prospective multicenter study is needed. Second, the selection bias is inevitable as only patients who underwent surgical resection were included. Therefore, more cases, including follow-up cases treated by chemotherapy or radiotherapy without surgical indication, should be included to determine the true accuracy of additional PET to CT. Third, the selection is based on surgeon's decision, which may cause selection bias.

In conclusion, additional PET has advantages over CT alone in clinically distinguishing benign from malignant tumors of the mediastinum, regardless of additional MRI. Furthermore, adding PET may help guiding the decision-making process for performing surgical resection for anterior mediastinal tumors.

\section{Acknowledgements}

Not applicable.

\section{Funding}

No funding was received.

\section{Availability of data and materials}

The datasets used and/or analyzed during the present study are available from the corresponding author upon reasonable request.

\section{Authors' contributions}

TY and AM were involved in the study design. TY contributed to the preparation of the manuscript and data analysis. EY, RO, TK, KS and HK contributed to the data analysis and prepared the tables. All authors read and approved the final manuscript and agree to be accountable for all aspects of the work.

\section{Ethics approval and consent to participate}

The present study was approved by the ethics committee of Gunma University Hospital. The requirement for informed consent was waived by the ethics committee because of the study's retrospective nature; however, patients could opt out of sharing their information.

\section{Patient consent for publication}

Not applicable.

\section{Competing interests}

The authors declare that they have no competing interests.

\section{References}

1. Duwe BV, Sterman DH and Musani AI: Tumors of the mediastinum. Chest 128: 2893-2909, 2005.

2. Yoneda KY, Louie S and Shelton DK: Mediastinal tumors. Curr Opin Pulm Med 7: 226-233, 2001.

3. Kern JA, Deniel TN, Trible CG, Silen ML and Rodgers BM: Thoracoscopic diagnosis and treatment of mediastinal masses. Ann Thorac Surg 56: 92-96, 1993.

4. Rendina EA, Venuta F, De Giacomo T, Ciriaco PP, Pescarmona EO, Francioni F, Pulsoni A, Malagnino F and Ricci C: Comparative merits of thoracoscopy, mediastinoscopy, and mediastinotomy for mediastinal biopsy. Ann Thorac Surg 57: 992-995, 1994.

5. David SE, Gregory JR, and Wallace A: NCCN Clinical Practice Guidelines in Oncology (NCCN Guidelines $\left.{ }^{\circledR}\right)$ Thymomas and Thymic Carcinomas. J Natl Compr Cancer Netw 11: 562-576, 2013.

6. Tomiyama N, Honda O, Tsubamoto M, Inoue A, Sumikawa H, Kuriyama K, Kusumoto M, Johkoh T and Nakamura H: Anterior mediastinal tumors: Diagnostic accuracy of CT and MRI. Eur J Radiol 69: 280-288, 2009. 
7. Sakai S, Murayama S, Soeda H, Matsuo Y, Ono M and Masuda K Differential diagnosis between thymoma and non-thymoma by dynamic MR imaging. Acta Radiol 43: 262-268, 2002.

8. Endo M, Nakagawa K, Ohde Y, Okumura T, Kondo H, Igawa S, Nakamura Y, Tsuya A, Murakami H, Takahashi T, et al: Utility of ${ }^{18}$ FDG-PET for differentiating the grade of malignancy in thymic epithelial tumors. Lung Cancer 61: 350-355, 2008.

9. Terzi A, Bertolaccini L, Rizzardi G, Luzzi L, Bianchi A, Campione A, Comino A and Biggi A: Usefulness of 18-F FDG PET/CT in the pre-treatment evaluation of thymic epithelial neoplasms. Lung Cancer 74: 239-243, 2011.

10. Lococo F, Cesario A, Okami J, Cardillo G, Cavuto S, Tokunaga T, Apolone G, Margaritora S and Granone P: Role of combined ${ }^{18} \mathrm{~F}-\mathrm{FDG}-\mathrm{PET} / \mathrm{CT}$ for predicting the WHO malignancy grade of thymic epithelial tumors: A multicenter analysis. Lung Cancer 82: 245-251, 2013.

11. Igai H, Matsuura N, Tarumi S, Chang SS, Misaki N, Go T, Ishikawa $\mathrm{S}$ and Yokomise $\mathrm{H}$ : Usefulness of $\left[{ }^{18} \mathrm{~F}\right]$ fluoro-2-deoxy-D-glucose positron emission tomography for predicting the world health organization malignancy grade of thymic epithelial tumors. Eur J Cardiothorac Surg 40: 143-145, 2011.

12. Otsuka H: The utility of FDG-PET in the diagnosis of thymic epithelial tumors. J Med Invest 59: 225-234, 2012.

13. Luzzi L, Campione A, Gorla A, Vassallo G, Bianchi A, Biggi A and Terzi A: Role of fluorine-flurodeoxyglucose positron emission tomography/computed tomography in preoperative assessment of anterior mediastinal masses. Eur J Cardiothorac Surg 36: 475-479, 2009.

14. Shibata H, Nomori H, Uno K, Sakaguchi K, Nakashima R, Iyama K, Tomiyoshi K, Kaji M, Goya T, Suzuki T and Horio H: ${ }^{18} \mathrm{~F}$-fluorodeoxyglucose and ${ }^{11} \mathrm{C}$-acetate positron emission tomography are useful modalities for diagnosing the histologic type of thymoma. Cancer 115: 2531-2538, 2009.

15. Kaira K, Sunaga N, Ishizuka T, Shimizu K and Yamamoto N: The role of $\left[{ }^{18} \mathrm{~F}\right]$ fluorodeoxyglucose positron emission tomography in thymic epithelial tumors. Cancer Imaging 11: 195-201, 2011.

16. Toba H, Kondo K, Sadohara Y, Otsuka H, Morimoto M, Kajiura K Nakagawa Y, Yoshida M, Kawakami Y, Takizawa H, et al: ${ }^{18} \mathrm{~F}$-fluorodeoxyglucose positron emission tomography/computed tomography and the relationship between fluorodeoxyglucose uptake and the expression of hypoxia-inducible factor- $1 \alpha$, glucose transporter-1 and vascular endothelial growth factor in thymic epithelial tumours. Eur J Cardiothorac Surg 44: e105-e112, 2013.
17. Fukumoto K, Taniguchi T, Ishikawa Y, Kawaguchi K, Fukui $\mathrm{T}$, Kato $\mathrm{K}$, Matsuo $\mathrm{K}$ and Yokoi $\mathrm{K}$ : The utility of $\left[{ }^{18} \mathrm{~F}\right]$-fluorodeoxyglucose positron emission tomography-computed tomography in thymic epithelial tumours. Eur J Cardiothorac Surg 42: e152-e156, 2012.

18. Sung YM, Lee KS, Kim BT, Choi JY, Shim YM and Yi CA: ${ }^{18}$ F-FDG PET/CT of thymic epithelial tumors: Usefulness for distinguishing and staging tumor subgroups. J Nucl Med 47: $1628-1634,2006$

19. Seki N, Sakamoto S, Karube Y, Oyaizu T, Ishihama H and Chida M: ${ }^{18} \mathrm{~F}$-fluorodeoxyglucose positron emission tomography for evaluation of thymic epithelial tumors: Utility for world health organization classification and predicting recurrence-free survival. Ann Nucl Med 28: 257-262, 2014

20. Yajima T, Mogi A, Shimizu K, Kosaka T, Ohtaki Y, Obayashi K, Nakazawa S, Nakajima T, Tsushima Y and Shirabe K: Quantitative analysis of metabolic parameters at ${ }^{18} \mathrm{~F}$-fluorodeoxyglucose positron emission tomography in predicting malignant potential of anterior mediastinal tumors. Oncol Lett 19: 1865-1871, 2020.

21. Kitami A, Sano F, Ohashi S, Suzuki K, Uematsu S, Suzuki T and Kadokura M: The usefulness of positron-emission tomography findings in the management of anterior mediastinal tumors. Ann Thorac Cardiovasc Surg 23: 26-30, 2017.

22. McErlean A, Huang J, Zabor EC, Moskowitz CS and Ginsberg MS: Distinguishing benign thymic lesions from early-stage thymic malignancies on computed tomography. J Thorac Oncol 8: 967-973, 2013.

23. Priola SM, Priola AM, Cardinale L, Perotto F and Fava C: The anterior mediastinum: Anatomy and imaging procedures. Radiol Med 111: 295-311, 2006.

24. Priola AM, Priola SM, Giraudo MT, Gned D, Fornari A, Ferrero B, Ducco L and Veltri A: Diffusion-weighted magnetic resonance imaging of thymoma: Ability of the apparent diffusion coefficient in predicting the world health organization (WHO) classification and the Masaoka-Koga staging system and its prognostic significance on disease-free survival. Eur Radiol 26: 2126-2138, 2016 